\title{
Programa de habilidades sociais para adolescentes em preparação para o trabalho
}

\author{
Camila de Sousa Pereira-Guizzo'; https://orcid.org/0000-0003-4197-8534 \\ Almir Del Prette²; https://orcid.org/0000-0003-2051-1214 \\ Zilda Aparecida Pereira Del Prette²; https://orcid.org/0000-0002-0130-2911 \\ Vanessa Barbosa Romera Leme ${ }^{3}$; https://orcid.org/0000-0002-9721-0439
}

\begin{abstract}
Resumo
Adolescentes de contextos socioeconômicos desfavoráveis têm menos acesso à educação e a boas condições de trabalho. Essa vulnerabilidade social exige dos adolescentes um repertório elaborado de habilidades sociais para enfrentar os desafios relacionados à inserção profissional e defesa de seus direitos. O objetivo deste estudo foi avaliar os efeitos de um Programa de Habilidades Sociais para superação de dificuldades interpessoais em diferentes situações do cotidiano de adolescentes que buscavam oportunidades de trabalho. Participaram 26 adolescentes (idade entre 14 e 16 anos) de baixa renda, distribuídos por meio de sorteio em dois grupos, sendo um de intervenção e outro de controle. Os instrumentos de avaliação foram: Inventário de Habilidades Sociais para Adolescentes e Diário de Campo. A intervenção mostrou efeito significativo na superação de dificuldades interpessoais relacionadas às habilidades sociais de Autocontrole e Abordagem social-sexual, consideradas importantes para a adaptação social, descoberta da sexualidade e convivência na escola ou no trabalho.
\end{abstract}

Palavras-chave: Habilidades sociais; avaliação de programa; adolescência.

\section{Social skills program for adolescents in preparing for work}

\begin{abstract}
Adolescents from unfavorable socio-economic backgrounds have less access to education and good working conditions. This social vulnerability requires the adolescents an elaborate repertoire of social skills to face the challenges related to the professional insertion and defense of their rights. The objective of this study was to evaluate the effects of a Social Skills Program to overcome interpersonal difficulties in different daily situations of adolescents who sought job opportunities. Twenty-six adolescents (age between 14 and 16 years) of low income participated, distributed through a lottery in two groups, one of intervention and one of control. The instruments of evaluation used were: Inventory of Social Skills for Adolescents and Field Diary. The intervention showed a significant effect on overcoming interpersonal difficulties related to the social skills of Self-control and Social-sexual Approach, considered important for social adaptation, discovery of sexuality and coexistence in school or work.
\end{abstract}

Key words: Social skills; program evaluation; adolescence.

\section{Programa de habilidades sociales para adolescentes en preparación para el trabajo}

\section{Resumen}

Adolescentes de contextos socioeconómicos desfavorables tienen menos acceso a la educación y a buenas condiciones de trabajo. Esa vulnerabilidad social exige de los adolescentes un repertorio elaborado de habilidades sociales para enfrentar los retos relacionados a la inserción profesional y defensa de sus derechos. El objetivo de este estudio fue evaluar los efectos de un Programa de Habilidades Sociales para superación de dificultades interpersonales en distintas situaciones del cotidiano de adolescentes que buscaban oportunidades de trabajo. Participaron 26 adolescentes (edad entre 14 y 16 años) de baja renta, distribuidos por intermedio de sorteo en dos grupos, siendo uno de intervención y otro de control. Los instrumentos de evaluación fueron: Inventario de Habilidades Sociales para Adolescentes y Diario de Campo. La intervención señaló efecto significativo en la superación de dificultades interpersonales relacionadas a las habilidades sociales de Autocontrol y Abordaje socialsexual, consideradas importantes para la adaptación social, descubierta de la sexualidad y convivencia en la escuela oen el trabajo.

Palabras clave: Habilidades sociales; evaluación de programas; adolescencia.

1 Centro Universitário SENAI CIMATEC - Salvador - BA - Brasil; camila.pereira@fieb.org.br

2 Universidade Federal de São Carlos - São Carlos - SP - Brasil; adprette@ufscar.br; zdprette@ufscar.br;

3 Universidade do Estado do Rio de Janeiro (UERJ). Rio de Janeiro - RJ - Brasil; vanessaromera@gmail.com 


\section{Introdução}

Por seu caráter de transição, a adolescência pode ser, muitas vezes, caracterizada como um momento de vida dominado pelo estresse e sentimentos ambíguos (Nightigale \& Fischooff, 2002). É verdade que um grande número de adolescentes é bem-sucedido na resolução das tarefas desenvolvimentais mais importantes dessa faixa etária, por exemplo, bom desempenho acadêmico, autonomia, relações próximas com pessoas do mesmo sexo e do sexo oposto e formação de identidade (Masten, 2014). Porém, a passagem a novos grupos e contextos sociais impõe aos adolescentes outras demandas interpessoais e torna mais provável sua exposição ao risco, como abuso de drogas lícitas e ilícitas, envolvimento com episódios de bullying e iniciação sexual precoce (Del Prette \& Del Prette, 2009a; Murta, Del Prette, \& Del Prette, 2010; Nightigale \& Fischooff, 2002). Por isso, as fases de transição do desenvolvimento podem ser consideradas como um período crítico para o indivíduo porque novas tarefas Ihes são exigidas, demandando habilidades sociais e adaptações para lidar com as mudanças, os desafios e as incertezas que se apresentam no curso de desenvolvimento (Del Prette \& Del Prette, 2009a; Fonseca, Sena, Santos, Dias, \& Costa, 2013; Pereira, Cia, \& Barham, 2008).

A transição para o mundo do trabalho durante a adolescência também tem recebido destaque na literatura psicológica brasileira (Amazarry, Thomé, Souza, \& Koller, 2009; Campos \& Francistini, 2003; Oliveira, Fischer, Teixeira, \& Amaral, 2003; Thomé, Pereira, \& Koller, 2016; Torres, Paula, Ferreira, \& Pinheiro, 2010). No Brasil, desde 2005, é permitido aos adolescentes exercerem atividade laboral como aprendizes. O Decreto $n^{\circ} 5.598$ (2005, art. $\left.3^{\circ}\right)$ admite que adolescentes com idade mínima de 14 anos possam ter "... a formação técnico-profissional metódica compatível com seu desenvolvimento físico, moral e psicológico...”. Todavia, deve-se ressaltar que o Estatuto da Criança e do Adolescente (ECA) proíbe que qualquer atividade de trabalho seja realizada por adolescentes menores de 14 anos.

Embora o Decreto $\mathrm{n}^{\circ} 5.598$ tenha surgido para combater os problemas gerados pela empregabilidade na adolescência, pesquisas indicam que as atividades profissionais podem, adicionalmente, dificultar o envolvimento dos adolescentes com as demandas escolares, as quais acabam sendo preteridas (Amazarry \& cols., 2009; Thomé \& cols., 2016; Torres \& cols., 2010). Em geral, as condições de trabalho oferecidas são precárias e competem com o desenvolvimento físico e socioemocional dos aprendizes (Oliveira \& cols., 2003). Contato precoce com a atividade laboral com relatos de insatisfação dos jovens trabalhadores também foi identificado na pesquisa de Torres e cols. (2010).

Alguns estudos identificaram que, na percepção dos pais de adolescentes de baixa renda, o trabalho tem o significado de proteger os filhos de um contexto de violência, marginalidade e tráfico de drogas (Alves-Mazzotti, 2002). Entretanto, o ambiente de trabalho, quando tem um caráter insalubre, também é nocivo para o desenvolvimento socioemocional dos adolescentes e, dessa forma, o que se verifica é uma manutenção do contexto de vulnerabilidade. A pesquisa realizada por Thomé e cols. (2016) é ilustrativa desse fato. Ao entrevistar 7425 adolescentes e jovens (idade entre 14 e 24 anos) de nível socioeconômico baixo, os autores encontraram que a maioria trabalhava em condições precárias, era mal remunerada e exercia a função laboral sem carteira assinada. Conforme argumentam alguns autores (Campos \& Francischini, 2003; Fonseca \& cols., 2013), muitas vezes o que leva os adolescentes das classes baixas a trabalhar é a condição de pobreza, a inexistência de políticas públicas de combate às desigualdades sociais e a ausência de escolarização integral.

Diante das possíveis situações de vulnerabilidade associadas à inserção dos adolescentes no mercado de trabalho, o Decreto $n^{\circ} 5.598$ especifica que o contrato deve ser como aprendiz e que a empresa deve possibilitar a conciliação entre trabalho e frequência escolar, além de promover a aprendizagem de habilidades específicas e competências profissionais. Desse modo, as experiências de trabalho na adolescência podem preparar para os desafios da vida adulta, como a escolha profissional e a construção de projeto de vida (Silva, 2016; Sousa, Frozzi, \& Bardagi, 2013).

Estudiosos que atuam com escolha profissional de adolescentes apontam que experiências com atividades laborais possibilitam o desenvolvimento das crenças de autoeficácia, autoestima mais elevada e melhor conhecimento sobre as próprias habilidades e requisitos para as diferentes profissões (Frenzel \& Bardagi, 2014; Silva \& Trindade, 2013; Silva, 2016; Sousa e cols., 2013). Portanto, as vivências estabelecidas com as atividades laborais também podem atuar positivamente para a aprendizagem social e a formação profissional, favorecendo algumas das tarefas desenvolvimentais dessa etapa de vida.

Assim, considerando a vulnerabilidade de adolescentes trabalhadores das camadas populares, mas também levando em consideração os aspectos positivos das experiências laborais na transição para a vida adulta, admite-se que a realização de um Programa de Habilidades Sociais (PHS) com adolescentes de nível socioeconômico baixo, em preparação para o trabalho, pode contribuir para um melhor aproveitamento da experiência. Essas habilidades podem auxiliar não só na escolha e inserção profissional, como também na defesa dos seus direitos e na reflexão da sua realidade caracterizada, muitas vezes, pela exploração da sua força de trabalho.

Para Del Prette e Del Prette (2017, p. 24), as habilidades sociais representam "um construto descritivo (1) dos comportamentos sociais valorizados em determinada cultura (2) com alta probabilidade de resultados favoráveis para o indivíduo, seu grupo e comunidade, (3) que podem contribuir para um desempenho socialmente competente em tarefas interpessoais". Del Prette e Del Prette (2009b) propõem seis classes interdependentes de habilidades sociais, relevantes para o relacionamento interpessoal satisfatório na adolescência: empatia; autocontrole; civilidade; assertividade; abordagem social-sexual; desenvoltura social. Um PHS é definido, por Del Prette e Del Prette (2010), como 
um conjunto de procedimentos e técnicas de intervenção que contemplam diversas atividades estruturadas, visando a favorecer a aprendizagem e/ou o desenvolvimento das habilidades sociais e, com isso, manter ou melhorar o relacionamento entre a pessoa e seus interlocutores (familiares, amigos, professores e parceiros românticos).

Ainda que sem focalizar a inserção no mercado de trabalho, PHS com adolescentes foram conduzidos tanto no contexto internacional (Kilian \& Kilian, 2011; Young \& cols., 2012) quanto nacional (Leme \& cols., 2016; Murta \& cols., 2012; Silva \& Murta, 2009) e evidenciaram que a aprendizagem das habilidades sociais de resolução de problemas (Kilian \& Kilian, 2011; Silva \& Murta, 2009), empatia, expressividade emocional e autocontrole, assertividade (Kilian \& Kilian, 2011; Leme \& cols., 2016; Murta \& cols., 2012) e abordagem social-sexual (Leme \& cols., 2016; Murta \& cols., 2010; Murta \& cols., 2012), contribuem para os adolescentes lidarem melhor com as demandas dessa etapa do ciclo vital, especialmente as afetivas e as assertivas ligadas a direitos sexuais e reprodutivos, diante da pressão do grupo de pares e no controle dos sentimentos.

Considerando o papel protetor das habilidades sociais, torna-se importante testar e aprimorar PHS para adolescentes em situação de vulnerabilidade. Além do empoderamento desses adolescentes para lidar com os desafios do contexto de trabalho, um programa desse tipo deve também ser capaz de promover a melhora dos seus relacionamentos com os familiares, pares, professores e parceiros românticos, bem como no exercício dos direitos humanos. Autores ainda ressaltam a necessidade de as escolas planejarem sistematicamente, em seus currículos, o aprimoramento das habilidades sociais e o fortalecimento da orientação profissional durante o percurso acadêmico de seus estudantes, a fim de reduzir problemas de comportamento, melhorar desempenho acadêmico e preparar os egressos para o mundo do trabalho (Pereira \& cols., 2008; Silva, 2016). Assim, esta pesquisa tem como objetivo analisar os efeitos de um PHS para a superação de dificuldades interpessoais em diferentes situações do cotidiano de adolescentes em condições de vulnerabilidade social e que buscam oportunidade de trabalho.

\section{Método}

\section{Participantes}

Participaram deste estudo 26 adolescentes de baixa renda (classe $\mathrm{C}$, segundo a pontuação média do Critério de Classificação Econômica Brasil - Associação Brasileira de Empresas de Pesquisa, 2003), com idade entre 14 e 16 anos $(M=14,4 ; d p=0,6)$. O estudo foi desenvolvido em uma Instituição Não-Governamental (ONG), localizada em uma cidade de médio porte do interior do estado de São Paulo. A instituição atende adolescentes em situações de risco social para a inclusão no mercado de trabalho por meio de diferentes ações, inclusive de formação de jovens aprendizes.
Foi realizada uma intervenção com amostra não-clínica, sob delineamento experimental, com avaliação pré e pós-teste. Os participantes foram distribuídos por meio de sorteio em dois grupos: (1) um que recebeu a intervenção e foi denominado de GE (Grupo Experimental); (2) o outro que ficou como Grupo Controle (GC) para atendimento posterior, ao término da intervenção com o GE. O GE foi composto por oito meninos e cinco meninas, com idade média de 14,6 anos $(d p=0,8)$, estudantes do $8^{\circ}$ ano do Ensino Fundamental (53,8\%), $1^{\circ}$ ano $(30,8 \%)$ e $2^{\circ}$ ano $(15,4 \%)$ do Ensino Médio. Já o GC foi composto por três meninos e 10 meninas, com idade média de 14,2 anos $(d p=0,4)$, sendo $92,3 \%$ alunos do $8^{\circ}$ ano do Ensino Fundamental e $7,7 \%$ do $1^{\circ}$ ano do Ensino Médio.

\section{Instrumentos}

Inventário de Habilidades Sociais para Adolescentes (IHSA-Del-Prette, Del Prette \& Del Prette, 2009b) - Instrumento de autorrelato para aferir a frequência e a dificuldade de adolescentes em emitir comportamentos socialmente habilidosos. Para este estudo, foi usada apenas a escala de dificuldade, que possui 38 itens, caracterizando diversas situações e diferentes interlocutores em uma escala tipo Likert de cinco pontos. O respondente assinala a sua dificuldade para apresentar a reação em uma das seguintes categorias: Nenhuma (0), Pouca (1), Média (2), Bastante (3), Total (4). $\mathrm{O}$ instrumento investiga habilidades sociais que são classificadas em seis fatores: (F1) Empatia, (F2) Autocontrole, (F3) Civilidade, (F4) Assertividade, (F5) Abordagem social-sexual e (F6) Desenvoltura social. O teste de Alpha de Cronbach, com as respostas aos 38 itens, dadas pelos participantes deste estudo, indicou que a escala tem elevada consistência interna $(a=0,86)$.

Diário de Campo - Caderno de registro para descrever e analisar eventos que ocorreram durante cada sessão da intervenção. O roteiro contribuiu para o registro de informações sobre: assiduidade, desempenho nas atividades e relatos de progressos.

\section{Considerações éticas}

Este estudo está de acordo com a Resolução $n^{\circ}$ 466/12 do Conselho Nacional de Saúde em relação às Diretrizes e Normas Regulamentadoras de Pesquisas Envolvendo Seres Humanos, tendo sido aprovado pelo Comitê de Ética (Parecer 95/06). Assim, após o contato e consentimento da instituição, os adolescentes foram convidados, em sala de aula, para participarem do estudo. A participação dos adolescentes foi voluntária e referendada pela entrega do Termo de Consentimento Livre e Esclarecido (TCLE) e do Termo de Assentimento Livre e Esclarecido (TALE), assinados pelos responsáveis legais e pelos adolescentes, respectivamente. 


\section{Procedimentos}

Coleta de dados. No primeiro contato, foram explicados os objetivos e os procedimentos do estudo para verificar se estavam dispostos a colaborar. O segundo encontro foi combinado para o recolhimento do TCLE e do TALE assinados e a aplicação coletiva do Inventário de Habilidades Sociais para Adolescentes (IHSA-Del-Prette). Nesse momento, os participantes receberam um código numérico, a fim de possibilitar o sorteio e a distribuição dos grupos. Houve também o cuidado de verificar se os grupos eram semelhantes nos escores de habilidades sociais antes da intervenção: encontrou-se diferença apenas no escore do fator Empatia, sugerindo dificuldade significativamente maior para o GE do que para o $\mathrm{GC}(U=45,5, p=0,04)$.

A instituição disponibilizou uma sala de aula adequada para a coleta de dados e a realização da intervenção. Após o término de cada sessão, a pesquisadora registrava a assiduidade, o desempenho nas atividades e os relatos de progressos no Diário de Campo. Após o encerramento da intervenção, foi aplicado novamente o IHSA-Del-Prette com ambos os grupos em horários e salas diferentes. Concluída a coleta de dados da pós-intervenção, o GC também recebeu a mesma intervenção realizada com o GE. Desde o princípio, ficou acordado que os participantes com pelo menos $75 \%$ de frequência na intervenção receberiam certificado pela participação. Também foi esclarecido que seria sorteado um brinde (livro) entre aqueles que não possuíam nenhuma falta nas sessões. O sorteio do livro ocorreu na última sessão. A entrega do certificado ocorreu ao final da coleta de dados.

O Programa de Habilidades Sociais. O objetivo da intervenção foi promover habilidades sociais para a superação de dificuldades interpessoais em diferentes situações do cotidiano, visando inclusive a preparação para o trabalho desses adolescentes. A intervenção foi em grupo e ocorreu em oito sessões, de aproximadamente 90 minutos de duração, distribuídas duas vezes por semana. Cada sessão foi dividida em três partes, como mostra a Figura 1.
$\mathrm{Na}$ parte central da sessão, os procedimentos de ensino de habilidades sociais foram promovidos essencialmente por meio das vivências. Vivências são definidas por Del Prette e Del Prette (2001, p. 106) como “.... atividade, estruturada de modo análogo ou simbólico a situações cotidianas de interação social dos participantes, que mobiliza sentimentos, pensamentos e ações, com objetivos de suprimir déficits e maximizar habilidades sociais em programas de THS em grupo". As vivências possibilitaram a aplicação de outros procedimentos como instrução, modelação, ensaio comportamental, videofeedback e tarefas de casa, os quais visaram superar os déficits apoiando-se nos recursos dos participantes, assim como atender a necessidades específicas que foram surgindo no decorrer das sessões.

A Tabela 1 descreve os objetivos e as vivências de cada sessão. Essas vivências foram descritas por Del Prette e Del Prette (2001), com detalhamento das atividades previstas em cada uma delas.

Conforme sugerido por Del Prette e Del Prette (2001), as sessões iniciais buscaram capacitar os participantes nas habilidades básicas para interações entre as pessoas nos vários contextos sociais. Essas habilidades básicas referem-se à observação e descrição de desempenhos sociais, discriminando componentes verbais e os componentes não-verbais na comunicação, oferecer feedbacks. Porém, essas habilidades continuaram sendo exercitadas e aprimoradas ao longo da intervenção por meio da participação e das discussões das vivências.

As sessões intermediárias focalizaram habilidades que atendiam às queixas dos participantes, relatadas durante a participação nas vivências (tais como, dificuldade de se expor em grupo, controlar raiva quando não aprova alguma situação, lidar com uma crítica) e que já haviam sido avaliadas por meio do IHSA-Del-Prette na pré-intervenção. As sessões intermediárias também prepararam os participantes para as habilidades mais complexas (defesa de direitos, assertividade, lidar com críticas) na medida em que facilitou a exposição e a coesão do grupo. Por isso, as habilidades

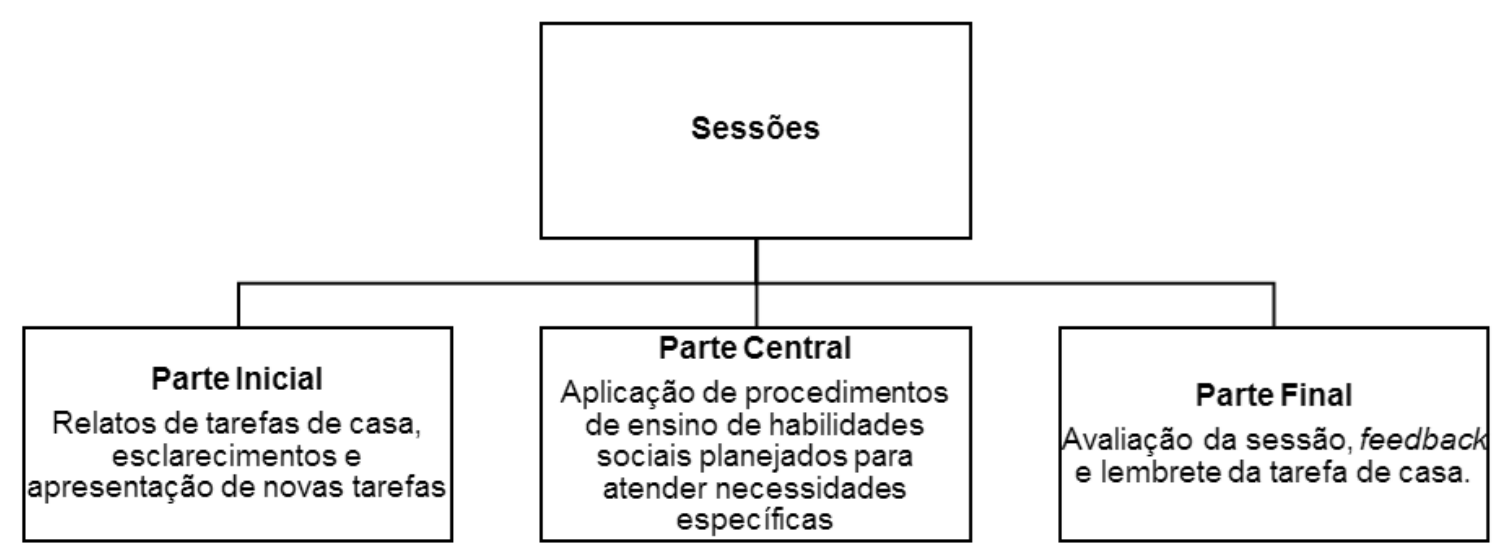

Figura 1. Estrutura das sessões. 
Tabela 1. Objetivos e vivências do PHS para adolescentes.

\begin{tabular}{|c|c|c|}
\hline Sessão & Objetivo & Vivência* \\
\hline $1^{a}$ & $\begin{array}{l}\text { Integrar os membros do grupo, conscientizando-os sobre a importância } \\
\text { do nome e do contato visual, fortalecendo a identidade pessoal e } \\
\text { profissional dos mesmos. } \\
\text { Observar e descrever desempenhos sociais, discriminando } \\
\text { componentes verbais de conteúdo, de forma e os componentes não- } \\
\text { verbais na comunicação. }\end{array}$ & $\begin{array}{l}\text { "O meu nome é..." } \\
\text { "Números Poéticos" }\end{array}$ \\
\hline $2^{\mathrm{a}}$ & $\begin{array}{l}\text { Compreender a importância do feedback para a correção, manutenção } \\
\text { e melhoria das relações interpessoais. } \\
\text { Expressar compreensão e sentimentos relacionados às dificuldades do } \\
\text { interlocutor. } \\
\text { Desenvolver uma comunicação empática. }\end{array}$ & "Vivendo o papel do outro" \\
\hline $3^{a}$ & $\begin{array}{l}\text { Compreender a importância do contato visual nas relações, além de } \\
\text { favorecer a oportunidade de manter contato visual com o interlocutor. } \\
\text { Iniciar e manter conversação. } \\
\text { Discriminar componentes não-verbais na comunicação. }\end{array}$ & "Olho nos olhos" \\
\hline $4^{a}$ & $\begin{array}{l}\text { Desenvolver habilidades de falar em público. } \\
\text { Aprimorar a fluência verbal, do improviso e da autoconfiança. } \\
\text { Exercitar o feedback. }\end{array}$ & "História coletiva oral" \\
\hline $5^{a}$ & $\begin{array}{l}\text { Identificar os direitos de adolescentes e trabalhadores. } \\
\text { Analisar a importância da relação pensamento-sentimento- } \\
\text { comportamento na defesa dos direitos. }\end{array}$ & $\begin{array}{l}\text { "Direitos humanos e } \\
\text { interpessoais" }\end{array}$ \\
\hline $6^{a}$ & $\begin{array}{l}\text { Identificar as diferenças entre o comportamento passivo, assertivo e } \\
\text { agressivo. } \\
\text { Refletir sobre os efeitos de tais comportamentos nas relações } \\
\text { interpessoais. } \\
\text { Observar e descrever desempenhos sociais. }\end{array}$ & $\begin{array}{l}\text { "Nem passivo nem } \\
\text { agressivo: assertivo!" }\end{array}$ \\
\hline $7^{a}$ & $\begin{array}{l}\text { Fazer críticas de modo socialmente competente. } \\
\text { Lidar com críticas justas e injustas. } \\
\text { Exercitar o feedback. }\end{array}$ & "Misto-quente" \\
\hline $8^{a}$ & $\begin{array}{l}\text { Conversar com pessoas de autoridade. } \\
\text { Desenvolver argumentação. } \\
\text { Desenvolver postura apropriada. }\end{array}$ & "Entrada no céu" \\
\hline
\end{tabular}

Fonte: *As vivências foram extraídas de Del Prette e Del Prette (2001).

mais complexas foram programadas para a fase final da intervenção.

Análise dos dados. Os dados obtidos com a versão de dificuldade do IHSA-Del-Prette foram computados em escore total e escores fatoriais. Os dados coletados antes e após a intervenção foram analisados no Statistical Package for the Social Sciences for Windows (SPSS, versão 22.0). Estatística não paramétrica foi utilizada para comparar os resultados das habilidades sociais entre os grupos, do seguinte modo: Mann-Whitney para comparar os resultados da pré e pós-intervenção entre GE e GC; Wilcoxon para comparar os resultados da pré e pós-intervenção no GE. Quanto ao Diário de Campo, os dados permitiram identificar a presença, por meio de registro de frequência, e ainda registrar o relato espontâneo de alguns participantes na íntegra.

\section{Resultados}

A assiduidade nas sessões é uma medida importante para o acompanhamento da avaliação de processo de uma intervenção. Na maioria das sessões houve $100 \%$ de presença. Apenas na sessão 4 e na sessão 8 ocorreram faltas, porém justificadas. Em relação à assiduidade por participante, quatro deles obtiveram $87,5 \%$ de presença e os demais obtiveram 100\%. A análise da presença por participante também indica poucas faltas. A proporção de adolescentes que participaram das sessões pode ser considerada como satisfatória, facilitando ao terapeuta boas condições para utilizar as vivências planejadas e que os participantes se beneficiassem do desenvolvimento promovido pelas atividades e discussões em grupo. 
Tabela 2. Análise Descritiva e de Comparação do Escore Total e dos Escores Fatoriais para o Grupo Experimental (GE, $n=13)$ e o Grupo Controle (GC, n=13) na Pós-Intervenção, para a Escala de Dificuldades

\begin{tabular}{|c|c|c|c|c|c|}
\hline Habilidades Sociais & Grupos & $\begin{array}{c}\text { Pré- } \\
\text { Mediana }\end{array}$ & $\begin{array}{c}\text { Pós- } \\
\text { Mediana }\end{array}$ & $\begin{array}{l}\text { Mann- } \\
\text { Whitney }\end{array}$ & $p$ \\
\hline \multirow{2}{*}{ Escore total } & GE & 59,0 & 44,0 & 110,5 & 0,18 \\
\hline & GC & 45,0 & 62,0 & & \\
\hline \multirow{2}{*}{ F1 - Empatia } & GE & 12,0 & 11,0 & 98,0 & 0,51 \\
\hline & GC & 7,0 & 10,0 & & \\
\hline \multirow{2}{*}{ F2 - Autocontrole } & GE & 16,0 & 14,0 & 127,0 & $0,02^{*}$ \\
\hline & GC & 15,0 & 19,0 & & \\
\hline \multirow{2}{*}{ F3 - Civilidade } & GE & 4,0 & 7,0 & 91,50 & 0,72 \\
\hline & GC & 3,0 & 7,0 & & \\
\hline \multirow{2}{*}{ F4 - Assertividade } & GE & 9,0 & 7,0 & 106,50 & 0,26 \\
\hline & GC & 7,0 & 9,0 & & \\
\hline \multirow{2}{*}{ F5 - Abordagem social-sexual } & GE & 11,0 & 11,0 & 112,50 & 0,15 \\
\hline & GC & 13,0 & 13,0 & & \\
\hline \multirow{2}{*}{ F6 - Desenvoltura social } & GE & 11,0 & 9,0 & 98,50 & 0,47 \\
\hline & GC & 6,0 & 9,0 & & \\
\hline
\end{tabular}

A Tabela 2 apresenta a análise descritiva do escore total e dos escores dos fatores das habilidades sociais e as diferenças entre o grupo experimental (GE) e o grupo controle (GC) na pós-intervenção, para a escala de dificuldades.

Na pós-intervenção, embora o GE tenha relatado dificuldades menores do que o GC no escore total e em alguns fatores, o teste Mann-Whitney aponta que o GE obteve diminuição significativa na escala de dificuldades com a intervenção no fator Autocontrole ( $U=127,0, p=0,029)$.

Importante analisar também os efeitos significativos no próprio grupo experimental. A Figura 2 compara as dife- renças dos resultados antes e depois da intervenção, exclusivamente, no GE.

Ao serem analisados os resultados pré e pós-intervenção nos participantes do GE, verificou-se que há diminuição significativa na escala de dificuldades, por meio do teste Wilcoxon, nos fatores F2 - Autocontrole $(z=-2,08, p=0,03)$ e F5 - Abordagem social-sexual ( $z=-2,05, p=0,04)$.

As anotações no Diário de Campo indicam que alguns adolescentes $(P)$ relataram espontaneamente a importância desse tipo de programa para a o desenvolvimento social: "O que eu mais gostei foi eu tentar interagir com todos através

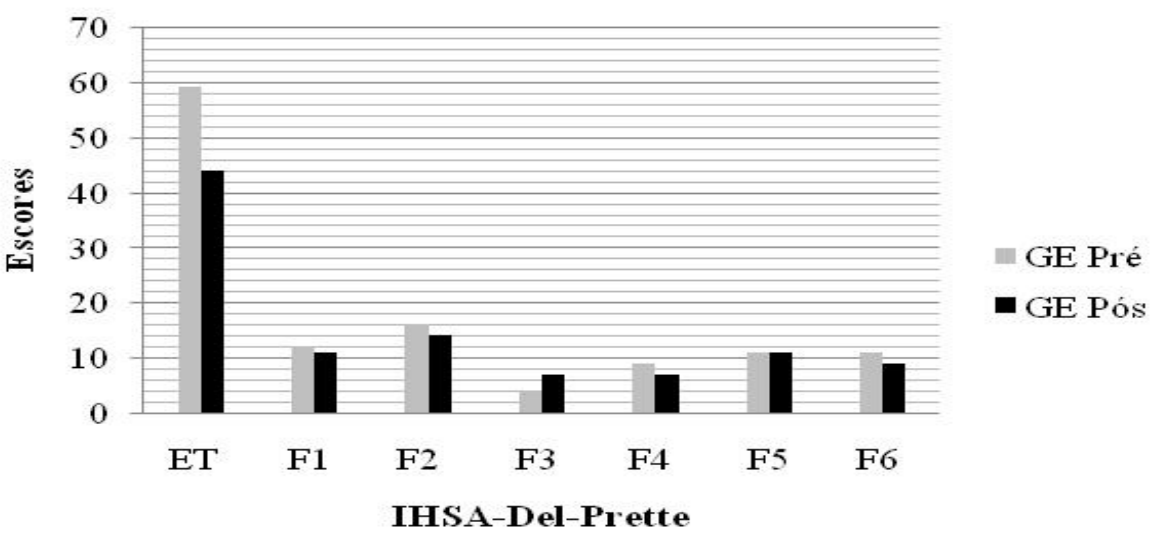

Figura 2. Diferença obtida (teste de Wilcoxon) na pré e pós-intervenção com o IHSA-Del-Prette no GE $(n=13)$, para a escala de dificuldades. Legenda: $\mathrm{ET}=$ Escore Total; F1 = Empatia; F2 = Autocontrole; F3 = Civilidade; F4 = Assertividade; F5 = Abordagem social-sexual; F6 = Desenvoltura social. 
das habilidades sociais (P3).", "O que mais gostei foi tudo! Mas o melhor foi que eu comecei a aceitar críticas e a lidar com ela." (P6), "Foi um treinamento muito legal que levaremos a vida toda em várias situações." (P1).

\section{Discussão}

Este estudo mostrou os efeitos de um PHS para superação de dificuldades interpessoais de adolescentes em condições de vulnerabilidade social e que buscam oportunidade de trabalho. A transição a novos grupos e contextos sociais, como o ambiente de trabalho, somando-se às condições insalubres de trabalho, problemas de relacionamento interpessoal e dificuldade de conciliar as demandas acadêmicas e profissionais são situações que aumentam a vulnerabilidade de adolescentes, podendo gerar comportamentos pouco adaptativos que, por sua vez, produzem resultados negativos para o desenvolvimento psicológico. Essas situações requerem um repertório aprimorado de habilidades sociais para lidar adequadamente com tais tarefas.

Primeiramente, discute-se a diferença inicial entre os grupos sinalizada na pré-intervenção. Essa análise mostrou que o fator Empatia foi avaliado significativamente mais difícil para o GE em comparação ao GC. Já na pós-intervenção (Tabela 2), verificou-se que a dificuldade relacionada ao fator Empatia reduziu para o grupo GE e aumentou para o GC. Assim, considerando que a presença inicial de maior dificuldade no GE pode gerar mais prejuízos na proficiência com que essa habilidade é emitida, uma vez que a dificuldade pode trazer maior custo de resposta (Del Prette \& Del Prette, 2010), nota-se que a intervenção contribuiu para a redução da dificuldade em relação à Empatia.

Os resultados destacaram redução estatisticamente significativa de dificuldades do GE nas habilidades relacionadas aos fatores Autocontrole e Abordagem social-sexual. Em relação ao fator Autocontrole, houve superação tanto na análise intergrupo como na intragrupo. Segundo Del Prette e Del Prette (2009b, p. 20), esse fator é definido como "habilidades de reagir com calma a situações aversivas em geral, tais como as que produzem sentimento de frustração, desconforto, raiva, humilhação". Os possíveis problemas gerados pela falta de autocontrole (como perda do emprego, agressões verbais ou físicas, exclusão social) podem registrar no adolescente experiências sociais de fracasso, exercendo influência negativa na formação do autoconceito e autoeficácia e, por sua vez, prejudicar o desenvolvimento psicológico e os relacionamentos interpessoais (Pereira \& cols., 2008).

Conforme Del Prette e Del Prette (2009b, p. 20), "não significa deixar de expressar desagrado ou raiva, mas fazêlo de forma socialmente competente, pelo menos em termos de controle sobre os próprios sentimentos negativos". Certamente, a ampliação de recursos pessoais para lidar com essas situações pode influenciar positivamente no enfrentamento de demandas específicas das relações de trabalho e de tarefas desenvolvimentais típicas dessa fase (adoção de novos papéis, aumento de responsabilidades, desempenho acadêmico e profissional), assim como ao longo de todo o ciclo de vida, prevenindo conflitos interpessoais e outras consequências desagradáveis.

Corroborando os achados de outros PHS com adolescentes (Leme \& cols., 2016; Murta \& cols., 2010; Murta \& cols., 2012), o fator Abordagem social-sexual, que "reúne as habilidades de estabelecer contato e conversação para relação sexual, para fazer amizades, para entrar em grupos da escola ou do trabalho, expressar satisfação ou insatisfação em relação a diferentes formas de carinho" (Del Prette \& Del Prette, 2009b, p. 20), obteve redução significativa de dificuldade na análise intragrupo. Considerando essas e outras demandas interpessoais e socioafetivas na adolescência, como adaptação às mudanças físicas, ampliação dos vínculos com o grupo de pares, início da vida sexual etc., pode-se notar a importância da aquisição das habilidades relacionadas à Abordagem social-sexual a fim de preparar os adolescentes para uma realidade que pode ser caracterizada tanto por experiências positivas como negativas, exigindo deles comportamentos socialmente habilidosos para facilitar a socialização, mas também preservar sua autoestima e seus direitos.

O envolvimento e as aquisições obtidas do GE com o PHS também foram sinalizados por meio de suas falas espontâneas. Não houve relatos de incompatibilidade de horário para as sessões de treinamento, nem de queixas quanto à dificuldade na realização das atividades, sugerindo que a estrutura e os procedimentos do PHS foram alternativas de intervenção viável para o desenvolvimento de adolescentes em preparação para o trabalho.

Quanto aos aspectos que contribuíram para a redução das dificuldades nos fatores Autocontrole e Abordagem social-sexual do GE, verificou-se a importância de planejar uma intervenção, definindo objetivos e procedimentos para cada sessão com base em resultados de uma avaliação pré-intervenção, considerando ainda as queixas e o contexto social dos participantes. Esses cuidados são sustentados pelas recomendações das práticas psicológicas baseadas em evidências (American Psychological Association, 2005) e do treinamento em habilidades sociais (Del Prette \& Del Prette, 2011).

Em relação ao fator Civilidade, o GE relatou aumento da dificuldade após a intervenção. Uma hipótese para esse resultado é que o ensino dessa habilidade não foi programado em nenhuma sessão especifica. Isso mostra a importância da programação de ensino, o que possibilita a definição dos objetivos comportamentais a serem ensinados e o arranjo de contingências de ensino-aprendizagem (Nale, 1998) a fim de que as habilidades sociais sejam de fato alvo de intervenção. Uma explicação alternativa seria considerar que o PHS possibilitou aumento do autoconhecimento dos adolescentes em relação à dificuldade de apresentar as habilidades sociais de Civilidade, indicando uma percepção subestimada na pré-intervenção.

Vale ainda comentar as mudanças de escores no GC. Essas mudanças sugeriram aumento de dificuldade, segundo relato do grupo controle, na maioria dos fatores 
avaliados pelo IHSA-Del-Prette. Uma possível explicação pode ser a expectativa do GC pela intervenção (que ocorria com o GE). A espera pode ter gerado no GC alguma ansiedade, inferida pela curiosidade em saber o que se passava e pela motivação em participar da intervenção, o que pode ter influenciado a segunda autoavaliação.

\section{Considerações Finais}

Este estudo mostrou que a intervenção promoveu superação de dificuldades interpessoais relacionadas, principalmente, às habilidades sociais de Autocontrole e Abordagem social-sexual. Além disso, os resultados sugerem o reconhecimento de que o PHS promoveu aprendizagem de habilidades sociais que ultrapassaram o âmbito profissional e envolveram diferentes situações sociais, conforme o objetivo do programa.

Algumas limitações devem ser consideradas. Primeiro, a aplicação de apenas um instrumento de avaliação de habilidades sociais e com apenas um indicador (dificuldade), bem como ter sido realizado com amostra pequena. Segundo, não foi realizada uma análise qualitativa dos relatos espontâneos dos participantes sobre a avaliação da intervenção.

Para o aprimoramento desse tipo de experimentação, novas pesquisas deveriam incrementar os instrumentos de avaliação utilizando, além do autorrelato, a observação direta, a avaliação por outros significantes (por exemplo, pares, cuidadores e professores), assim como incluir a escala de frequência de habilidades sociais para análise do déficit de desempenho.

Novos estudos também poderiam realizar entrevistas para obter relatos sobre a avaliação do processo e dos efeitos da intervenção, bem como acompanhar a manutenção do aprendizado e a efetividade desse tipo de intervenção para a inclusão no mercado de trabalho. A replicação da intervenção em larga escala também é uma recomendação importante para a contribuição na produção de conhecimento, o que pode levar à adoção e à disseminação desse programa em diferentes serviços ou organizações.

\section{Referências}

Alves-Mazzotti, A. J. (2002). Repensando algumas questões sobre o trabalho infanto-juvenil. Revista Brasileira de Educação, 19(1), 87-98.

Amazarray, M. R.; Thomé, L. D.; Souza, A. P. L.; Polleto, M.; Koller, S. (2009). Aprendiz versus Trabalhador: Adolescentes em processo de aprendizagem. Psicologia: Teoria e Pesquisa, 25(30), 329-338.

American Psychological Association (2005). Report of the 2005 presidential task force on evidence-based practice. Washington, DC: Autor.
Associação Brasileira de Empresas de Pesquisa (2003). Critério de Classificação Econômica Brasil. São Paulo: Autor.

Campos, H. R.; Francischini, R. (2003). Trabalho infantil produtivo e desenvolvimento humano. Psicologia em Estudo, 8(1), 119-129.

Decreto $n^{\circ} 5.598$ de 1 de dezembro de 2005 (2005, $1^{\circ}$ de dezembro). Regulamenta a contratação de aprendizes e dá outras providências. Brasília: Presidência da República.

Del Prette, A.; Del Prette, Z. A. P. (2001). Psicologia das relações interpessoais: Vivências para o trabalho em grupo. Petrópolis, RJ: Vozes.

Del Prette, A.; Del Prette, Z. A. P. (2009a). Adolescência e fatores de risco: A importância das habilidades sociais educativas. In: Haase, V. G.; Ferreira, F. O.; Penna, F. J. (Orgs.), Aspectos biopsicossociais da saúde na infância e adolescência (pp. 503522). Belo Horizonte: Coopmed.

Del Prette, A.; Del Prette, Z. A. P. (2009b). Inventário de habilidades sociais para adolescentes (IHSA-Del-Prette): Manual de aplicação, apuração e interpretação. São Paulo: Casa do Psicólogo.

Del Prette, A.; Del Prette, Z. A. P. (2017). Competência Social e Habilidades Sociais: Manual teórico-prático. Petrópolis: Vozes.

Del Prette, Z. A. P.; Del Prette, A. (2010). Habilidades Sociais e análise do comportamento: Proximidade histórica e atualidades. Perspectivas em Análise do Comportamento, 1(2), 38-49.

Del Prette, Z. A. P.; Del Prette, A. (2011). Práticas baseadas em evidência e treinamento de habilidades sociais. In: Del Prette, A.; Del Prette, Z. (Orgs.), Habilidades sociais: Intervenções efetivas em grupo (pp. 57-76). São Paulo: Casa do Psicólogo.

Fonseca, F. F.; Sena, R. K. R.; Santos, R. L. A.; Dias, O. V.; Costa, S. M. (2013). The vulnerabilities in childhood and adolescence the brazilian public policy intervention. Revista Paulista de Pediatria, 31(2), 258-64.

Frenzel, H. S.; Bardagi, M. P. (2014). Adolescentes trabalhadores brasileiros: Um breve estudo bibliométrico. Revista Psicologia, 14(1), 79-88.

Kilian, J. M.; Kilian, D. W. (2011). A school intervention to increase prosocial behavior and improve academic performance of at-risk students. Improving Schools, 14(1), 65-83.

Leme, V. B. R.; Fernandes, L. M.; Jovarini, N. V.; El Achkar, A. M.; Del Prette, Z. A. P. (2016). Social Skills Program for adolescents in vulnerable social contexts. Psico-USF, 21(3), 598-608.

Masten, A. S. (2014). Global Perspectives on Resilience in Children and Youth. Child Development, 85(1), 6-20.

Murta, S. G.; Del Prette, A.; Del Prette, Z. A. P. (2010). Prevenção 
ao Sexismo e ao heterossexismo entre adolescentes. Revista de Psicologia da Criança e do Adolescente, 2, 73-86.

Murta, S. G.; Ribeiro, D. C.; Rosa, I. O.; Menezes, J. C. L.; Rieiro, M. R. S.; Borges, O. S.; Paulo, S. G.; Oliveira, V.; Miranda, V. H.; Del Prette, A.; Del Prette, Z. A. P. (2012). Programa de habilidades interpessoais e direitos sexuais e reprodutivos para adolescentes: Um relato de experiência. Psico-USF, 17(1), 21-32.

Nale, N. (1998). Programação de ensino no Brasil: O papel de Carolina Bori. Revista de Psicologia da USP, 9, 275-301.

Nightingale, E. O.; Fischhoff, B. (2002). Adolescent risk and vulnerability: Overview. Journal of Adolescent Health, 31(1), 3-9.

Oliveira, D. C.; Fischer, F. M.; Teixeira, M. C. T. V. (2003). A escola e o trabalho entre adolescentes do ensino médio da cidade de São Paulo: Uma análise das representações sociais. Psicologia: Teoria e Pesquisa, 5(1), 27-39.

Pereira, C. S.; Cia, F.; Barham, E. J. (2008). Autoconceito, habilidades sociais, problemas de comportamento e desempenho acadêmico na puberdade: Inter-relações e diferenças entre sexos. Interação em Psicologia, 12(2), 203-213.
Silva, L. (2016). Estudo sobre a orientação vocacional e profissional Escolhas. Psicologia Escolar e Educacional, 20(2), 239-244.

Silva, M. P.; Murta, S. G. (2009). Treinamento de habilidades sociais para adolescentes: Uma experiência no Programa de Atenção Integral à Família. Psicologia: Reflexão e Crítica, 22(1), 136-143.

Silva, R. D. M.; Trindade, Z. A. (2013). Adolescentes aprendizes: Aspectos da inserção profissional e mudanças na percepção de si. Revista Brasileira de Orientação Profissional, 14(1), 73- 86.

Sousa, H.; Frozzi, D.; Bardagi, M. P. (2013). Perceptions of adolescent apprentices about the experience of first job. Psicologia: Ciência e Profissão, 33(4), 918-933.

Thomé, L. D.; Pereira, A. S.; Koller, S. H. (2016). O desafio de conciliar trabalho e escola: Características sociodemográficas de jovens trabalhadores e não-trabalhadores. Psicologia: Teoria e Pesquisa, 32(1), 101-109.

Torres, C. A.; Paula, P. H. A.; Ferreira, A. G. N.; Pinheiro, P. N. C. (2010). Adolescência e trabalho: Significados, dificuldades e repercussões na saúde. Interface: Comunicação, Saúde e Educação, 14(35), 839-850.

Young, J. F.; Makover, H. B.; Cohen, J. R.; Mufson, L.; Gallop, R. J.; Benas, J. S. (2012). Interpersonal psychotherapy-adolescent skills training Anxiety outcomes and impact of comorbidity. Journal of Clinical Child \& Adolescent Psychology, 41(5), 640-653.

Recebido: 18 de setembro de 2017 Aceito: 16 de fevereiro de 2018

Apoio: Fundação de Amparo à Pesquisa do Estado de São Paulo (FAPESP) unrestricted use, distribution and reproduction in any medium, provided the original article is properly cited. 